\title{
Standardized Questionnaire for the Measurement, Valuation, and Estimation of Costs of Informal Care Based on the Opportunity Cost and Proxy Good Method
}

\author{
Erik Landfeldt $^{1,2}$ - Niklas Zethraeus ${ }^{3} \cdot$ Peter Lindgren $^{3}$
}

Published online: 13 August 2018

(c) The Author(s) 2018

\begin{abstract}
Costs of informal care account for a significant component of total societal costs for many chronic and disabling illnesses. Yet, costs associated with the provision of informal care is seldom included in economic evaluations of new health technologies, increasing the risk of suboptimal decisions on the allocation of resources from the perspective of society. Our objective was to propose a standardized questionnaire for the measurement, valuation, and estimation of caregiver indirect (productivity) and informal care costs as separate mutually exclusive subsets of total costs in cost-of-illness studies and as an input to economic evaluations from the societal perspective. We developed a questionnaire for data collection and step-by-step analysis procedures for resource valuation and cost estimation. Data concerning absenteeism from work and time devoted to informal care were recorded using the recall method. Indirect (productivity) and paid informal care costs were valued and estimated according to the human-capital approach as the loss of production. Unpaid informal care costs were valued and estimated as the loss of leisure time quantified using the opportunity cost and proxy good method. The new questionnaire, titled the Caregiver Indirect and Informal Care Cost Assessment Questionnaire, contains 13 questions regarding caregiver current and previous work status, productivity, and the provision of informal care (stratified by time devoted to household activities, personal care, practical support, and emotional support). The proposed questionnaire should be helpful to inform the design, implementation, and execution of future cost-of-illness studies and economic evaluations from the perspective of society.
\end{abstract}

Electronic supplementary material The online version of this article (https://doi.org/10.1007/s40258-018-0418-2) contains supplementary material, which is available to authorized users.

Erik Landfeldt

erik.landfeldt@ki.se

1 Institute of Environmental Medicine, Karolinska Institutet, Nobels väg 13, 17177 Stockholm, Sweden

2 Icon plc, Stockholm, Sweden

3 Department of Learning, Informatics, Management and Ethics, Medical Management Centre, Karolinska Institutet, Stockholm, Sweden

\section{Key Points}

From the perspective of society, informal caregiving may be associated with non-trivial costs as a result of absenteeism from work and lost leisure time.

For some diseases, omitting costs associated with informal care may result in suboptimal decisions on the allocation of resources from the perspective of society.

We propose a new standardized questionnaire for the estimation of caregiver indirect (productivity) and informal care costs from the perspective of society.

\section{Introduction}

Informal caregiving (i.e., care provided by non-professional unpaid individuals, usually family members, other relatives, or close friends, outside of the licensed 
or certified formal care sector) constitutes an important substitute for and complement to formal care. In fact, it has been estimated that informal care accounts for up to $90 \%$ of the in-home long-term care needed by adults in USA [1]. As a consequence, in addition to adverse health effects for the individual caregiver, informal caregiving is associated with substantial costs from the perspective of society, as parents, partners, friends, and other relatives reduce their working hours, stop working completely, and/ or devote a significant proportion of their leisure time to provide informal care [2]. Indeed, recently published recommendations from the Second Panel on Cost-Effectiveness in Health and Medicine underscore the importance of including implications of informal care in economic evaluations conducted from the societal perspective [3].

However, despite its prevalence and magnitude in many indications, costs associated with caregiver burden have historically seldom been included in economic evaluations, which would be expected to result in suboptimal policy decisions and inefficient allocation of healthcare resources from the perspective of society $[4,5]$. Aside from differences in the perspective of analysis adopted by national reimbursement agencies, a potential reason for the absence of these costs in health technology assessments is the lack of robust and relevant data, as estimating caregiver indirect costs (also known as productivity costs, which refer to the loss of production as a result of absenteeism from work from the perspective of society [3]) and informal care costs (which refer to costs associated with the provision of care outside of the formal healthcare sector) requires the collection and analysis of information on many different aspects of work status and leisure time [6]. Moreover, in studies aiming to measure, value, and estimate caregiver indirect and informal care costs as separate mutually exclusive subsets of total costs, which is usually necessary for inclusion in economic evaluations, particular care is required to avoid double counting.

Yet, although a wide array of tools has been developed to measure different elements of the caregiver burden [2, $7,8]$, to our knowledge, no instrument has been proposed specifically for the collection of data and valuation and estimation of caregiver indirect and informal care costs. In addition, the lack of a standardized tool to assess costs associated with informal care also limits the possibilities to conduct meaningful comparisons of estimates across studies. The objective of this article is to present a questionnaire for the measurement, valuation, and estimation of caregiver indirect (productivity) and informal care costs as separate mutually exclusive subsets of total costs in cost-of-illness studies and as an input to economic evaluations from the societal perspective.

\section{Methods}

\subsection{Measuring, Valuing, and Estimating Indirect (Productivity) Costs}

In the field of health economics, in contrast to other research disciplines, indirect cost has become synonymous with the monetary value of lost production as a result of absenteeism from work or reduced productivity $[9,10]$. In brief, two main methods for the estimation of indirect (productivity) costs exist: (1) the human-capital approach, and (2) the friction cost method. The traditional and most commonly applied method for estimating indirect costs in economic evaluations and cost-of-illness studies is the human-capital approach, in which the loss in production is quantified as the number of lost work hours valued at the cost of employment (i.e., the national mean gross wage plus employer's costs and social fees), that is, the opportunity cost of labor. The human-capital approach is also the most widely designated method for the estimation of indirect costs for use in economic evaluations as per guidelines from reimbursement agencies that employ a societal perspective in their evaluations [11], such as the Dental and Pharmaceutical Benefits Agency in Sweden [12].

According to the friction cost method, production losses occur only during the time it takes to replace a worker, i.e., the friction period [13]. Although preferred by health technology assessment bodies in some countries, e.g., the Netherlands [11], the friction cost method has been shown to be based on implausible assumptions not supported by neoclassical economic theory [14]. Moreover, for implementation, the friction cost method relies on micro-level labor market information, which limits its usefulness in settings in which such data are not readily available. Comparing the human-capital approach and the friction cost method, by design, cost estimates using the former will be higher (as they comprise production losses accumulated for a longer duration of time). Recently, an alternative for estimating costs of productivity losses for employers based on detailed information from managers and the derivation of wage multipliers has been proposed in the literature [15]. However, analogously to the friction cost method, the applicability of this method, despite generating accurate estimates, would be expected to be limited by non-trivial micro-level data requirements.

\subsection{Measuring, Valuing, and Estimating Informal Care Costs}

To estimate informal care costs, which for the purpose of the present study refer specifically to the time the caregiver 
spends providing informal care (not e.g., out-of-pocket expenses or other costs associated with the formal or informal care of the patient carried out by the caregiver, or the monetary value of impaired health-related quality of life), it is necessary to record the time devoted to informal care activities and tasks (e.g., helping with dressing, preparing food and feeding, accompanying to doctors' appointments, and providing emotional support). Two main methods for the measurement of such data are described in the literature: (1) the diary method, and (2) the recall method [2]. In the former, the caregiver is asked to register all the time spent on caregiving during a specific timeframe (e.g., a day), usually stratified by activity/task. In the latter method, the caregiver is asked to specify the time spent on informal care in general and/or for specific care activities and tasks during a specific timeframe (e.g., a day or week). In practice, most cost research utilizes the recall method, possibly as it is considered too burdensome for caregivers to keep a diary of their tasks, but also as such detailed data in our experience is seldom needed for the estimation of costs, in particular when considering the granularity of the other resource data typically collected in cost-of-illness studies.

Once measured, the time spent providing informal care can be valued (i.e., converted to monetary units) in a number of ways. The two most commonly applied methods include the proxy good method (also known as the replacement cost approach) [16] and the opportunity cost method [17]. In the former, the time recorded for the different activities and tasks are valued at a shadow price of a market substitute (e.g., a housekeeper for housekeeping services and a nurse for nursing services). In other words, using the proxy good method, informal caregiving activities and tasks are considered and valued as work, as opposed to lost leisure time. In contrast, using the opportunity cost method, informal care time is instead valued at the opportunity cost from the perspective of the caregiver. This is usually based on the individual's wage, but can also include estimates derived using other techniques (e.g., conjoint analysis, contingent valuation, or estimates of the value of travel time savings, which represent the monetary value of reduced travel time).

Although a full review of the strengths and weaknesses of the proxy good and opportunity cost methods is outside the scope of this article, it is worth mentioning that, from a theoretical point of view, the latter method is preferred because resources (and time inputs) should be valued at their opportunity costs to society [17]. Moreover, compared with the opportunity cost method, an advantage of the proxy good method is that different caregiver tasks can be valued separately, resulting in potentially more precise cost estimates. A drawback is that the method fails to incorporate differences in quality and efficiency between formal and informal care, which may also have an impact on patient health-related quality of life. In turn, a key disadvantage with the opportunity cost method concerns valuation and the need to identify the opportunity cost of leisure time [16].

\subsection{Standardized Questionnaire for the Measurement, Valuation, and Estimation of Caregiver Indirect (Productivity) and Informal Care Costs}

As noted in Sect. 1, estimating caregiver indirect (productivity) and informal care costs as separate subsets of total costs from the perspective of society can be challenging as the analysis comprises lost work time (including lost productivity while working), as well as paid and unpaid informal care. To that end, in this article, we propose a new standardized questionnaire that explicitly measures, values, and estimates these different cost components separately. The questionnaire is designed to be generic and thus applicable irrespective of the disease or condition of the patient or geographical setting. In addition, Stata, SAS, and R analysis procedures for the step-by-step valuation and estimation of indirect and informal care costs based on the data recorded by the questionnaire are provided as Electronic Supplementary Material (ESM).

The specification of the new questionnaire was based on: (1) data requirements for the measurement, valuation, and estimation of caregiver indirect (productivity) and informal care costs according to the human-capital approach, the proxy good method, and the opportunity cost method; (2) our previous experience of developing case report forms for cost-of-illness research across different indications and therapeutic areas; and (3) a targeted literature review of previous frameworks and tools for estimating caregiver indirect and informal care costs (details provided as ESM).

The questionnaire was designed to measure, value, and estimate caregiver indirect (productivity) costs according to the human-capital approach. To that end, we devised questions to record data on all relevant aspects of previous and current work status (including employment status, work hours, absenteeism, and productivity while working) to measure the total loss of work hours. To estimate informal care costs, we formulated questions to record the number of hours of leisure time (i.e., non-working hours) devoted to informal care using the recall method.

To allow a more precise valuation of informal care based on the proxy good method, we included four different categories of informal care activities and tasks, of which three were modified versions of questions included in the iMTA Valuation of Informal Care Questionnaire [18] (a previously developed measure of different aspects of informal care): (1) household activities, (2) personal care, (3) practical support, and (4) emotional support. As specified in the iMTA Valuation of Informal Care Questionnaire, in an attempt to only 
record the additional time spent on household activities and similar tasks of caregiving associated with the disease or condition of the patient, the questions relating to the provision of informal care included an explanation that only activities and tasks that the caregiver would not have had to perform if the patient was in good health, or if she/he could have done them, should be considered. We chose to not measure time devoted to non-caregiving and caregiving household activities and tasks separately, as previous research has shown that such a distinction may result in an underestimation of the time devoted to informal caregiving [19].

In addition, the questionnaire was formulated to also record data concerning paid informal care, that is, the time (if any) that the caregiver is financially compensated (e.g., by the state) to care for the patient. Adjustment for joint production (i.e., when an individual performs several activities at the same time or during a specific period of time) was not considered as there is evidence that respondents account for this when reporting time using the recall method [20].

In the standardized questionnaire and the accompanied analysis procedures, two different approaches were implemented depending on the choice of valuation of informal care. Specifically, using the proxy good method, recorded hours of informal care were accounted for in full as an informal care cost, whereas only the cost associated with lost work hours (for caregivers who reduced their working hours or stopped working completely) that were not substituted by informal care were accounted for, and subsequently valued, as an indirect (productivity) cost. This is because informal care, using the proxy good method, is regarded as work from the perspective of society. Put differently, using this method, the estimated cost associated with informal care is subtracted from the estimated indirect cost.

In the second approach, based on the opportunity cost method, informal care cost was estimated analogously, whereas indirect (productivity) cost was accounted for in full. This is because informal care, using the opportunity cost method, is valued and estimated in terms of lost leisure time from the perspective of the caregiver, and thus not regarded as work. It should be noted that there is evidence from previous research that caregivers may overestimate the time they devote to informal care, and for this reason, some studies have imposed a constraint to allow the caregiver time for basic needs (e.g., eating, sleeping, and toileting) [2]. We included this option in our analysis procedures. Last, using both methods, paid informal care was measured as work hours, valued at the cost of employment.

\section{Results}

\subsection{Caregiver Indirect and Informal Care Cost Assessment Questionnaire}

The new questionnaire, titled the Caregiver Indirect and Informal Care Cost Assessment Questionnaire (CIIQ), is shown in Fig. 1. It contains 13 questions concerning caregiver work status and the provision of paid and unpaid informal care. Tables 1 and 2 present the different steps involved in the estimation of caregiver indirect (productivity) and informal care costs of illness based on the data collected using the CIIQ. The described analysis procedure for indirect costs encompasses a total of six steps, differentiating between caregivers who are employed full-time, employed part-time, and unemployed. The procedures for estimating informal care costs include three steps, differentiating between paid and unpaid informal care. Stata, SAS, and R analysis procedures for the step-by-step estimation of indirect and informal care costs are provided as ESM.

\subsection{Example Applications}

To showcase the instrument, we now present example calculations for three fictitious US caregivers with different work status and involvement in the provision of paid and unpaid informal care. In these examples, to obtain annual estimates, it is assumed that the mean number of working weeks per year in USA is 48 [21]. For the calculation of indirect (productivity) costs, we assumed that that the cost of employment (i.e., the gross wage plus employer's costs and social fees) is US $\$ 35$ per hour [22]. For the valuation of informal care using the opportunity cost method, we assumed a gross wage of US\$20 for the opportunity cost of work time, and $35 \%$ of the gross wage $(0.35 \times$ US $\$ 20=$ US\$7) for the opportunity cost of leisure time, the latter in line with recently updated estimates of the value of travel time savings [23].

Using the proxy good method, we assumed for simplicity that the market substitute cost of all informal care activities and tasks (i.e., household activities, personal care, practical support, and emotional support) was US\$50 per hour. Additionally, in our example analyses, we imposed a minimum amount of leisure time of $6 \mathrm{~h}$ per day to allow the caregiver time for basic needs. Accordingly, the maximum amount of time per week available for work, informal care, and leisure was $(24-6) \times 7=126 \mathrm{~h}$. However, it is important to note that all these assumptions can be easily modified by the researcher using the CIIQ. 
Fig. 1 Caregiver Indirect and Informal Care Cost Assessment Questionnaire (CIIQ)

The Caregiver Indirect and Informal Care Cost Assessment Questionnaire

We will now ask you questions regarding your current work status and the time that you spend caring for your child, partner, relative, or friend. Please note that we from now on will refer to the person you care for as "relative", although your relationship may be different.

\section{WORK STATUS}

1 Are you currently employed or self-employed (working for pay)?

Yes

No $\rightarrow$ (skip to question 8$)$

2 How many hours per week do you work (e.g. according to your employment contract)? hours per week

3 Are you currently working full-time?

Yes $\rightarrow$ (skip to question 6 )

No

4 Did you reduce your working hours due to your relative's disease/condition (e.g. to care for him/her)?

Yes

No $\rightarrow$ (skip to question 6$)$

5 How many hours per week did you work before reducing your working hours? hours per week

6 During the last week, how many hours did you miss from work due to your relative's disease/condition?

Include hours missed when you came in late or left work early because you e.g. accompanied your relative to doctor appointments, visited hospitals or clinics, or helped your relative dressing, grooming, eating, or take medications.

hours per week

7 During the last week, how much did your relative's disease/condition affect your productivity while you were working?

If you were able to work as usual, choose a low number. If you were not able to work as usual (e.g. accomplished less than usual, could not concentrate or perform certain tasks as carefully as usual), choose a high number.

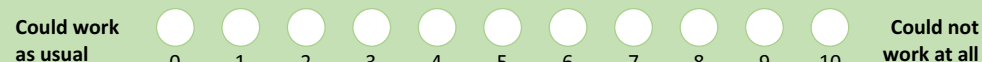

$\begin{array}{lllllllllllll}\text { as usual } & 0 & 1 & 2 & 3 & 4 & 5 & 6 & 7 & 8 & 9 & 10 & \text { work at all }\end{array}$

$\rightarrow$ Go to question 9

8 If not working: Did you stop working because of your relative's disease/condition?

Yes $\rightarrow$ How many hours per week did you used to work? $\quad$ hours

No

9 Are you compensated in any way for the time that you care for your relative? This include payment from e.g. the government or an insurance company to care for your relative.

Yes $\rightarrow$ How many hours per week are you paid to care for your relative? hours

No 
Fig. 1 (continued)

The Caregiver Indirect and Informal Care Cost Assessment Questionnaire

INFORMAL CAREGIVING

10 During the last week, how much time did you spend on household activities and tasks that you would not have had to perform if your relative was in good health, or if she/he could have done them independently?

For example preparing food, cleaning, washing, ironing, sewing, shopping, and gardening.

hours per week

11. During the last week, how much time did you spend helping your relative with her/his personal care?

For example dressing/undressing, washing/showering/bathing, hair care, shaving and grooming, and going to the toilet.

hours per week

12 During the last week, how much time did you spend providing practical support to your relative that would not have had to be performed if she/he were in good health, or if she/he could have done it independently?

For example eating and drinking, moving inside or outside the house (including assistance with walking or using a wheelchair), visiting family or friends, accompany to healthcare visits (e.g. doctor appointments), filling prescriptions at the pharmacy, help taking medications, and taking care of financial matters (e.g. paying the bills or managing healthcare insurance).

hours per week

13 During the last week, how much time did you spend on providing emotional support to your relative that would not have had to be provided if she/he were in good health? For example help to cope with pain, disability, and discomfort, anxiety, and worry.

hours per week 
Table 1 Steps to estimate caregiver indirect (productivity) costs of illness using the Caregiver Indirect and Informal Care Cost Assessment Questionnaire

\section{Step 1 Calculate total work hours including paid informal care}

The first step to estimate caregiver indirect (productivity) costs is to calculate the total number of work hours per week, including hours that the caregivers are paid to care for their relative, using data recorded in Question 2 and Question 9

\section{Step 2 Calculate the loss of work hours while working}

The second step is to estimate the loss of work hours while working (for caregivers who are employed). For this calculation, the recorded loss in productivity from Question 7 is transformed to a proportion representing percent work impairment/loss. This estimate is then multiplied by the number of work hours per week (recorded in Question 2) adjusted for the number of hours missed from work (recorded in Question 6). The calculated loss is then added to the number of hours missed from work (recorded in Question 6), which represents the total loss of work hours for caregivers employed full-time

\section{Step 3 Calculate the loss of work hours for caregivers employed part-time}

The third step is to calculate the loss of work hours for caregivers who are employed part-time (i.e., No on Question 3). This should only comprise hours for caregivers who state that they reduced their working hours because of the disease/condition of the relative (i.e., Yes on Question 4). To calculate the loss of work hours, total work hours (calculated in Step 1) are subtracted from previous work hours (recorded in Question 5). This loss is then added to the number of hours missed while working (calculated in Step 2)

Step 4 Calculate the loss of work hours for caregivers not employed

The fourth step is to estimate the loss of work hours for caregivers who are unemployed (i.e., No on Question 1). This calculation should only comprise caregivers who state that they stopped working because of the disease/condition of the relative (i.e., Yes on Question 8). To calculate the loss of work hours, the number of hours of paid informal care recorded in Question 9 is subtracted from previous work hours recorded in Question 8

\section{Step 5 Extrapolation}

The fifth step is to extrapolate the weekly data to annual estimates using external data on the country-specific mean number of work weeks per year

\section{Step 6 Valuation and estimation}

The sixth and final step is to multiply the estimated total annual loss of work hours with the cost of employment. Using the proxy good method, the value of informal care (calculated in Table 2) must be subtracted from the total value of lost work hours (excluding costs associated with loss of work hours owing to lost productivity while working calculated in Step 2)

Table 2 Steps to estimate caregiver informal care cost of illness using the Caregiver Indirect and Informal Care Cost Assessment Questionnaire

\section{Step 1 Calculate total number of hours of paid informal care}

The first step to estimate caregiver informal care cost is to calculate the number of hours of paid informal care per week. These data are recorded in Question 9. To obtain the annual number of hours of paid informal care, the weekly data are multiplied by 52, assuming

that the care is provided throughout the year

\section{Step 2 Calculate total number of hours of unpaid informal care}

The second step is to calculate the total number of hours of unpaid informal care per week. These data are recorded in Question 10 through Question 13. To obtain the annual number of hours of unpaid informal care, the weekly data are multiplied by 52, assuming that the informal care is provided throughout the year. The calculated number of hours of unpaid informal care may be adjusted to allow for a minimum time for basic needs (e.g., eating, sleeping, and toileting) of $6 \mathrm{~h}$ per day

\section{Step 3 Valuation and estimation}

Opportunity cost method

The third and last step to calculate informal care cost is to multiply the calculated hours of unpaid informal care by the chosen opportunity cost of leisure time (e.g., 35\% of the national mean gross wage according to the value of travel time savings [22]). Paid informal care (calculated in Step 1) is valued at the cost of employment

Proxy good method

The third and last step to calculate informal care cost is to multiply the calculated hours of unpaid informal care by the chosen market substitute costs. Paid informal care (calculated in Step 1) is valued at the cost of employment

\subsubsection{Example Caregiver A: Alice}

Alice works $40 \mathrm{~h}$ full-time, but is also paid $10 \mathrm{~h}$ per week to care for her son with a musculoskeletal condition. During the last week, Alice estimates that she lost $2 \mathrm{~h}$ while working (while attending a doctor appointment with her son), but rated her productivity loss at 0 out of 10 (i.e., that she could perform her tasks as usual while at work). In addition, she spends on average $12 \mathrm{~h}$ per week after work to care for her son. According to these data, Alice's total loss of work hours during the last week would be 2. Assuming that she works 48 weeks per year, her total annual loss would be $2 \times 48=96 \mathrm{~h}$, which implies a total annual indirect cost of $96 \times$ US $\$ 35=$ US $\$ 3360$. Moreover, her total 
annual number of hours of paid informal care would be $10 \times 52=520$, valued at $520 \times$ US $\$ 35=$ US $\$ 18,200$. Using the opportunity cost method, Alice's total annual number of hours of unpaid informal care would be $12 \times 52=624$, valued at $624 \times \mathrm{US} \$ 20 \times 0.35=\mathrm{US} \$ 4368$. Alternatively, using the proxy good method, Alice's total annual number of hours of unpaid informal care would also be 624, valued at $624 \times$ US $\$ 50=U S \$ 31,200$, and her total annual indirect cost would be US\$0 (because US\$3360 - US $\$ 31,200<$ US $\$ 0$ ) because she substitutes her lost work hours with informal care.

Thus, the total cost of Alice's informal care was estimated at US $\$ 18,200+\mathrm{US} \$ 4368=\mathrm{US} \$ 22,568$ and US $\$ 18,200+\mathrm{U}$ $S \$ 31,200=$ US $\$ 49,400$ using the opportunity cost and proxy good method, respectively. Last, we note that her total number of work, paid informal, and unpaid informal care hours do not exceed $126 \mathrm{~h}$ per week.

\subsubsection{Example Caregiver B: Brian}

Brian used to work full-time $35 \mathrm{~h}$ per week but stopped working completely when his wife was diagnosed with cancer. Instead, he spends on average $63 \mathrm{~h}$ per week caring for his wife at home (but is not paid to do so). Accordingly, Brian's total loss of work hours during the last week would be 35 , equal to $35 \times 48=1680 \mathrm{~h}$ per year, which implies a total annual indirect cost of $1680 \times$ US $\$ 35=\mathrm{US} \$ 58,800$.

Moreover, using the opportunity cost method, his total annual number of hours of unpaid informal care would be $63 \times 52=3276$, valued at $3276 \times$ US $\$ 20 \times 0.35=\mathrm{US} \$ 22$, 932. Alternatively, using the proxy good method, Brian's total annual number of hours of unpaid informal care would also be 3276 , valued at $3276 \times$ US $\$ 50=U S \$ 163,800$, and his total annual indirect cost would be US $\$ 0$ (because US $\$ 58,800$ - US $\$ 163,800<$ US\$0).

In both cases, Brian's paid informal care cost would be US\$0 (as he was not paid to provide informal care). Thus, the total cost of Brian's informal care was estimated at US $\$ 22,932$ and US $\$ 163,800$ using the opportunity cost and proxy good method, respectively, and we note that his total number of work, paid informal, and unpaid informal care hours do not exceed $126 \mathrm{~h}$ per week.

\subsubsection{Example Caregiver C: Charlotte}

Charlotte reduced her working hours from $40 \mathrm{~h}$ to $20 \mathrm{~h}$ per week to help care for her sister with breast cancer. During the last week, Charlotte missed 2 work hours as a result of leaving work early, and rated her productivity loss at 5 out of 10 because she worried a lot about the prognosis of her sister and was therefore not able to pursue her work tasks as usual. She also reports spending $15 \mathrm{~h}$ per day caring for her sister. Accordingly, Charlotte's total loss of work hours during the last week would be $40-20=20$, which is equal to $20 \times 48=960 \mathrm{~h}$ per year, which implies a total annual indirect cost of $960 \times$ US $\$ 35=$ US $\$ 33,600$. In addition, she missed 2 work hours as a result of leaving work early, which is equal to $2 \times 48=96 \mathrm{~h}$ per year, valued at $96 \times \mathrm{US} \$ 35=\mathrm{US} \$ 3360$, and lost $(20-2) \times 0.50=9 \mathrm{~h}$ per week because of reduced productivity while working (recorded at 50\%), which equals $9 \times 48=432 \mathrm{~h}$ per year, valued at $432 \times$ US $\$ 35=$ US $\$ 15,120$. Consequently, Charlotte's total annual indirect cost would be US $\$ 33,600+$ US $\$ 3360$ $+\mathrm{US} \$ 15,120=\mathrm{US} \$ 52,080$.

Moreover, using the opportunity cost method, her total annual number of hours of unpaid informal care would be $15 \times 52=780$, valued at $780 \times$ US $\$ 20 \times 0.35=\mathrm{US} \$ 5460$. Alternatively, using the proxy good method, Charlotte's total annual number of hours of unpaid informal care would also be 780 , valued at $780 \times \mathrm{US} \$ 50=\mathrm{US} \$ 39,000$, and her total annual indirect cost would be US $\$ 0$ (because US $\$ 33,600+$ $\$ 3360$ - US\$39,000<US\$0).

In both cases, Charlotte's paid informal care cost would be US\$0 (as she was not paid to provide informal care). Thus, the total cost of Charlotte's informal care was estimated at US\$5460 and US $\$ 39,000$ using the opportunity cost and proxy good method, respectively, and we note that her total number of work, paid informal, and unpaid informal care hours do not exceed $126 \mathrm{~h}$ per week.

\section{Discussion}

The aim of this article was to present a new standardized questionnaire for the measurement, valuation, and estimation of caregiver indirect (productivity) and informal care costs as separate mutually exclusive subsets of total costs in costof-illness studies and as an input to economic evaluations from the societal perspective. Given the amount of information typically captured in cost-of-illness research, including data on healthcare resource use (e.g., hospital admissions, visits to physicians and other healthcare professionals, medical tests and assessments, medications, and emergency and respite care), non-medical resources (e.g., non-medical aids, devices, and investments, and transportation services), and patient work status, we sought to develop a short tool recording data of sufficient granularity while minimizing the burden on the respondents.

The proposed questionnaire, titled the CIIQ, was designed to measure all data needed to value and estimate these cost components, irrespective of the disease or condition of the patient or geographical setting. Despite being relatively brief, our example calculations show that the framework successfully accommodates analysis of caregivers with widely different work statuses and caregiving roles, including varied levels of absenteeism from work, lost productivity 
while working, and unpaid and paid informal care. Yet, with respect to the CIIQ, there are several important assumptions and limitations that warrant further discussion.

First, when extrapolating weekly or daily data to yearly estimates, the proposed questionnaire relies on external information on the mean number of weeks working per year (that is, a full calendar year minus the country-specific total number of weeks or days of annual leave). We chose this approach as we found it to be unfeasible to measure these data in the CIIQ (e.g., by asking caregivers how many weeks per year they work, or their total number of weeks of annual leave per year, which includes paid vacation days and public holidays). Although using external data may bias the cost estimates derived from the CIIQ, as the sample size increases, the mean number of weeks of annual leave in a given cohort of caregivers would be expected to converge to the mean for the total national population, as caregivers are not limited to some subgroups (as defined by e.g., indication or socio-economic status) of the general population. In fact, in USA, an estimated 65.7 million individuals, roughly $20 \%$ of the total population, were involved in the provision of informal care to children or adult patients in 2009 [1].

Second, it is worth noting that our proposed questionnaire only accommodates the estimation of costs associated with one caregiver (e.g., a single parent or partner). However, in many cases, in particular for childhood diseases, several individuals may contribute to the provision of informal care. Thus, the CIIQ may be complemented with additional questions regarding e.g., the proportion of informal care provided by the participating caregiver to allow for an estimation of total indirect and informal care costs.

Third, an important statistical consideration of the present study concerns information bias, a systematic error that arises from measurement error. The two main sources of information bias in our work are recall bias and incorrect reporting. Specifically, caregivers may find it difficult to precisely remember the time devoted to informal care or the number of hours of lost work time. In addition, caregivers may not be able to fully differentiate between the time spent on normal and informal care tasks [2]. We tried to alleviate the impact of this limitation by specifying recall periods in accordance with conventional specifications/descriptions of work hours in general media and employment agreements (e.g., number of work hours employed per week, as opposed to per month or year). For hours of unpaid informal care, we chose to measure the daily number of hours to minimize recall bias. Yet, to further limit incorrect reporting, depending on the target indication and geographical setting, we suggest including help texts, as well as logical tests and skip patterns if administered online, to ensure that the recorded data are accurate and complete.

Fourth, for extrapolation, the CIIQ analysis procedures assumes that the provision of informal care is uniformly distributed across the year for any given patient. Although this would be expected to be a reasonable simplification for chronic health states, for acute symptoms such as stroke or short-term injuries, this assumption could result in an under- or overestimation of the informal caregiving burden (depending on the timing of the informal care).

Fifth, and last, it is important to emphasize that more research is needed to understand the psychometric properties of the CIIQ as applied in populations of caregivers, including test-retest reliability. Validation studies, in which the information recorded in the CIIQ is cross-checked with historical data on absenteeism as collated in population-based administrative databases, would be helpful to further assess the robustness of the CIIQ.

\section{Conclusions}

We propose a new standardized questionnaire, the CIIQ, for the measurement, valuation, and estimation of caregiver indirect (productivity) and informal care costs as separate mutually exclusive subsets of total costs in cost-of-illness studies and as an input to economic evaluations from the societal perspective. The CIIQ should be helpful to inform the design, implementation, and execution of future cost studies encompassing resources beyond those directly attributable to the medical care of the patient, and facilitate economic evaluations from the perspective of society.

Acknowledgements The authors gracefully acknowledge Dr. Matthias Hunger, Icon plc, Munich, Germany, for implementing the analysis procedures in SAS and R.

Author Contributions Erik Landfeldt originated the idea for the study. Erik Landfeldt led the development of the questionnaire with input from Peter Lindgren and Niklas Zethraeus. Erik Landfeldt developed the analysis procedures and drafted the manuscript. Peter Lindgren and Niklas Zethraeus revised the manuscript for important intellectual content. All authors approved the decision to submit for publication. Erik Landfeldt is the overall guarantor of the article.

\section{Compliance with Ethical Standards}

Funding No sources of funding were received for the preparation of this article.

Conflict of interest Erik Landfeldt, Peter Lindgren, and Niklas Zethraeus have no conflicts of interest directly relevant to the contents of this article.

Open Access This article is distributed under the terms of the Creative Commons Attribution-NonCommercial 4.0 International License (http://creativecommons.org/licenses/by-nc/4.0/), which permits any noncommercial use, distribution, and reproduction in any medium, provided you give appropriate credit to the original author(s) and the 
source, provide a link to the Creative Commons license, and indicate if changes were made.

\section{References}

1. Adelman RD, Tmanova LL, Delgado D, Dion S, Lachs MS. Caregiver burden: a clinical review. JAMA. 2014;311:1052-60.

2. Hoefman RJ, van Exel J, Brouwer W. How to include informal care in economic evaluations. Pharmacoeconomics. 2013;31(12):1105-19.

3. Sanders GD, Neumann PJ, Basu A, et al. Recommendations for conduct, methodological practices, and reporting of cost-effectiveness analyses: second Panel on Cost-Effectiveness in Health and Medicine. JAMA. 2016;316(10):1093-103.

4. Krol M, Papenburg J, van Exel J. Does including informal care in economic evaluations matter? A systematic review of inclusion and impact of informal care in cost-effectiveness studies. Pharmacoeconomics. 2015;33:123-35.

5. Krol M, Papenburg J, Koopmanschap M, Brouwer W. Do productivity costs matter? The impact of including productivity costs on the incremental costs of interventions targeted at depressive disorders. Pharmacoeconomics. 2011;29(7):601-19.

6. van den Berg B, Brouwer W, van Exel J, et al. Economic valuation of informal care: lessons from the application of the opportunity costs and proxy good methods. Soc Sci Med. 2006;62(4):835-45.

7. Mosquera I, Vergara I, Larrañaga I, et al. Measuring the impact of informal elderly caregiving: a systematic review of tools. Qual Life Res. 2016;25(5):1059-92.

8. Wimo A1, Jonsson L, Zbrozek A. The Resource Utilization in Dementia (RUD) instrument is valid for assessing informal care time in community-living patients with dementia. J Nutr Health Aging. 2010;14(8):685-90.

9. Johannesson M. The concept of cost in the economic evaluation of health care: a theoretical inquiry. Int J Technol Assess Health Care. 1994;10(4):675-82.

10. Drummond M, Sculpher M, Torrance G, et al. Methods for economic evaluation of health care programmes. 4th ed. Oxford: Oxford University Press; 2015.

11. International Society for Pharmacoeconomics and Outcomes Research. Pharmacoeconomic guidelines around the world. http:// www.ispor.org/PEguidelines/index.asp. Accessed 5 May 2017.
12. The Dental and Pharmaceutical Benefits Agency. General guidelines for economic evaluations from the Pharmaceutical Benefits Board (LFNAR 2003:2). http://www.tlv.se/Upload/English/Guide lines-for-economic-evaluations-LFNAR-2003-2.pdf. Accessed 1 Jun 2017.

13. Koopmanschap M, Rutten F, Vanineveld B, Vanroijen L. The friction cost method for measuring indirect costs of disease. J Health Econ. 1995; 14:171-89.

14. Johannesson M, Karlsson G. The friction cost method: a comment. J Health Econ. 1997;16(2):249-55.

15. Strömberg C, Aboagye E, Hagberg J. Estimating the effect and economic impact of absenteeism, presenteeism, and work environment-related problems on reductions in productivity from a managerial perspective. Value Health. 2017;20(8):1058-64.

16. Koopmanschap MA, van Exel JN, van den Berg B, Brouwer WB. An overview of methods and applications to value informal care in economic evaluations of healthcare. Pharmacoeconomics. 2008;26:269-80.

17. Posnett J, Jan S. Indirect cost in economic evaluation: the opportunity cost of unpaid inputs. Health Econ. 1996;5(1):13-23.

18. The iMTA Valuation of Informal Care Questionnaire (iVICQ). https://www.imta.nl/questionnaires. Accessed 9 May 2017.

19. van den Berg B, Spauwen P. Measurement of informal care: an empirical study into the valid measurement of time spent on informal caregiving. Health Econ. 2006;15(5):447-60.

20. Robinson JP. The validity and reliability of diaries versus alternative time use measures. In: Juster FT, Stafford FP, editors. Time, goods, and well-being. Ann Arbor (MI): The University of Michigan Institute for Social Research; 1985.

21. Van Giezen R. Paid leave in private industry over the past 20 years. Beyond the numbers: pay and benefits. US Bureau of Labor Statistics, 2013. http://www.bls.gov/opub/btn/volume-2/paid-leave in-private-industry-over-the-past-20-years.htm. Accessed 10 May 2017.

22. US Bureau of Labor Statistics. https://www.bls.gov/fls/country/ united_states.htm. Accessed 1 June 2017.

23. US Department of Transportation. The value of travel time savings: departmental guidance for conducting economic evaluations, revision 2 (2011). http://www.dot.gov/sites/dot.dev/files/docs/ vot_guidance_092811c.pdf. Accessed 1 Jun 2017. 